STUDI

FRANCESI

\section{Studi Francesi}

Rivista quadrimestrale fondata da Franco Simone

185 (LXII | II) | 2018

OCTAVE MIRBEAU: UNE CONSCIENCE AU TOURNANT

DU SIEECLE - sous la direction de Ida Merello

\title{
Octave Mirbeau, un canonisateur à canoniser
}

\section{Marjana Djukic}

\section{(2) OpenEdition \\ Journals}

Édition électronique

URL : http://journals.openedition.org/studifrancesi/12609

DOI : 10.4000/studifrancesi. 12609

ISSN : 2421-5856

Éditeur

Rosenberg \& Sellier

\section{Édition imprimée}

Date de publication : 1 août 2018

Pagination : 259-267

ISSN : 0039-2944

\section{Référence électronique}

Marjana Djukic, « Octave Mirbeau, un canonisateur à canoniser », Studi Francesi [En ligne], 185 (LXII |

II) | 2018, mis en ligne le 01 septembre 2019, consulté le 09 janvier 2021. URL : http://

journals.openedition.org/studifrancesi/12609; DOI : https://doi.org/10.4000/studifrancesi. 12609

\section{(2) $(\mathcal{Q} \Theta \Theta$}

Studi Francesi è distribuita con Licenza Creative Commons Attribuzione - Non commerciale - Non opere derivate 4.0 Internazionale. 


\title{
Octave Mirbeau, un canonisateur à canoniser
}

\begin{abstract}
Journalistic criticism that evaluates contemporary authors often falls into the sin of ephemeral judgments with no value to the literary history. The example of Octave Mirbeau is quite exceptional because the texts which he has published in newspapers reveal a critic of art whose assessments are valid, accepted and transmitted through literary and art histories.

This research examines Mirbeau's critical method in the context of the nineteenth century French criticism and discovers a unique approach where daily activity, sense of form, creative empathy and strong judgments intertwine and coexist. According to the classification of Thibaudet, Mirbeau unites all three types of criticism: journalistic, artists' and academic. However, this shows that the historical consciousness of Mirbeau reveals him as a latent artistic and literary historian. Thanks to his critical talent and evaluations, many authors of unquestionable status in today's histories are revealed by Octave Mirbeau. They include both writers and artists, such as: the Belgian authors Maurice Maeterlinck and Georges Rodenbach, but also Rodin, Monet, Camille Claudel. In his work on canonization Octave Mirbeau also proves himself to be a historian of art and literature by using the form of journalistic criticism. His judgments have been used by both historians and critics until today.
\end{abstract}

Pour situer la méthode critique d'Octave Mirbeau, initialement et superficiellement, nous nous référons à la classification célèbre de Thibaudet ${ }^{1}$ selon laquelle il existe trois types de critiques: la critique spontanée ou journalistique, la critique des professeurs et la critique des créateurs, des écrivains. Avec son expérience de critique journalistique de plus de quarante ans, on serait tenté de qualifier la critique d'Octave Mirbeau de journalistique. Mais, par son œuvre littéraire, originale et exceptionnelle, il appartient à la critique des écrivains aussi et nous ne pouvons pas négliger cet esprit empathique et créateur uni à l'esprit critique qui analyse et juge l'art. Sa méthode critique est tout à fait unique, ce que nous allons montrer ultérieurement, et elle a pour effet d'évaluer, de juger et de canoniser les auteurs contemporains, le plus souvent inconnus.

\section{La tradition critique}

Mirbeau utilise pleinement ce que nous appelons aujourd'hui la méthode externe, qui lie le texte analysé au contexte social. Il découvre aux lecteurs des éléments biographiques de l'auteur, ses impressions personnelles, les anecdotes et les rumeurs, il analyse même l'aspect physique comme dans le cas de Knut Hamsun:

Physionomie d'expression double, énergique et tendre, ardente et contenue, pénétrante et voilée, fière et triste, et, marquée, çà et là, aux joues creuses, aux narines pincées et reniflantes, des signes de la souffrance, elle impressionne et retient longtemps l'esprit'.

(1) A. Thibaudet, Physiologie de la critique, Paris, La Nouvelle Revue critique, 1930

(2) O. Mirbeau, Les Ecrivains, deuxième série, Paris, Flammarion, 1926, pp. 31-38. 
Cependant ces données factuelles ne servent pas à expliquer le texte ni à rendre un jugement. C'est plutôt un ornement rhétorique de la critique journalistique pour révéler toute une vie artistique au public, pour offrir un texte amusant et informatif en même temps, pour attirer l'attention sur la qualité concrète de l'art. Ce procédé de portraitiste, il en use principalement pour les auteurs inconnus ou étrangers. Même l'absence de ces informations devient dans le texte de Mirbeau un fait éloquent:

Je ne sais rien de M. Maurice Maeterlinck. Je ne sais d'où il est, ni comment il est. S'il est vieux ou jeune, riche ou pauvre, je ne le sais. Je sais seulement qu'aucun homme n'est plus inconnu que lui; et je sais aussi qu'il a fait un chef-d'œuvre'.

L'esprit fin d'Octave Mirbeau est conscient des limites de la critique biographique bien qu'il s'en serve pleinement. Il reproche à Sainte-Beuve le manque de créativité pour pouvoir juger et analyser ses contemporains. Mirbeau médite tout le temps sur la place des artistes dans l'histoire littéraire. Pour déterminer l'importance d'un auteur, il ne se borne pas au seul moment de la parution d'un livre, il situe son auteur dans la hiérarchie littéraire aussi bien du passé que du futur, tout en mettant l'accent sur la vision de l'avenir. Pour Mirbeau, la littérature est vivante, réelle, concrète, nouvelle et originale aussi bien que les artistes qu'il estime. Dans l'article $L a$ Postérité, où il aborde des sujets importants pour la critique, il décrit à sa manière le résultat du travail critique de Sainte-Beuve:

Ni une générosité, ni une bravoure et l'instinctive horreur du vrai, du simple, du vivant et la peur du nouveau. Jamais il ne s'intéressa spontanément à un jeune talent. Il fallait pour qu'il parlât des écrivains, que ceux-ci fussent morts depuis deux siècles ou qu'ils lui arrivassent vieillis de succès ${ }^{4}$.

Le péché mortel de la critique française au XIX ${ }^{e}$ siècle, malgré sa renommée incontestable, était l'ignorance de grands auteurs contemporains: ne citons que quelques exemples légendaires: Stendhal, Flaubert et surtout Baudelaire. Les célèbres critiques, de Sainte-Beuve à Taine et à Brunetière, restent les fondateurs de la critique littéraire moderne; ils ont élaboré des méthodes et des systèmes théoriques et critiques qui donnent des résultats brillants dans l'analyse des auteurs anciens mais qui se montrent moins utiles dans l'évaluation des auteurs contemporains. C'est la raison pour laquelle Mirbeau admire chez Hennequin «cette méthode hardie et vaste» qu'il applique «à l'étude d'hommes comme Victor Hugo, Wagner, Flaubert, Tolstoï, Dickens» ${ }^{5}$. L'esprit positiviste n'est pas étranger à Mirbeau: «Il avait donc élargi jusqu'à la science, reculé jusqu'à la création le champ rétréci de la critique»${ }^{6}$.

Octave Mirbeau est un critique averti qui engage sans cesse, implicitement ou explicitement, des polémiques avec les figures imposantes de la critique française. Ce travail, que nous appelons aujourd'hui métacritique, esquisse en grande partie son idéal critique. Parmi tous ces noms célèbres (Lemaitre, A. France, Brunetière), il privilégie le critique Gustave Geffroy. Dans l'article publié dans l'«Écho de Paris» en 1892, Mirbeau présente son livre La Vie artistique en mettant en valeur le style et la méthode de Geffroy:

(3) O. Mirbeau, Les Écrivains, première série, Paris, Flammarion, 1926, p. 174.

(4) Ibid., pp. 99-108.

(5) Ibid., pp. 117-126.

(6) Ibid. 
C'est toujours avec la même hauteur de pensées, avec le même profond regard qui va, pardelà les surfaces des couleurs et des formes, chercher l'âme même des choses et des êtres, c'est avec la même émotion de poète et de penseur que M. Gustave Geffroy nous montre les œuvres de Monet, de Rodin, de Pissarro, de Renoir, et qu'il les fait passer, de l'esprit même de ces admirables artistes, dans son esprit à lui, où elles gardent leur grandeur expliquée et comprise ${ }^{\top}$.

Mirbeau apprécie dans la méthode de Geffroy spécialement le fait qu'il est non seulement un critique bien informé, «mais un véritable constructeur de formes, un créateur d'idées au même degré que ceux dont il nous fait comprendre le génie» ${ }^{8}$. Dans le goût des formes, dans la recherche de l'âme des choses et des êtres, dans la reconnaissance du génie, Mirbeau se révèle artiste, créateur. L'espace de la critique journalistique devient étroit pour lui, son regard est plus profond et plus analytique. Sa pensée s'adresse à l'histoire, et seulement provisoirement au public du jour.

\section{La critique mirbellienne}

Les études récentes consacrées à la critique "d'auteur" soulignent le lien entre la domination de l'histoire littéraire "officielle" et la réaction des auteurs qui veulent penser l'histoire littéraire. Dans l'Introduction du livre L'Histoire littéraire des écrivains, qui définit et décrit l'histoire des créateurs, on trouve sa localisation dans le temps.

Si l'histoire littéraire des écrivains est partout présente à partir de la fin du XIX siècle, c'est qu'elle est bien souvent une réaction à l'apparition et à l'essor de l'histoire littéraire comme discipline. On peut la considérer comme une tentative de réappropriation, par les écrivains, d'une aventure qui est aussi (quoique pas seulement) la leur. Dans le même geste, cette tentative vaut d'ailleurs comme une reconnaissance de l'empire de l'histoire sur l'idée même de littérature?.

Les auteurs de cette Histoire estiment que les créateurs déplorent «une sorte de cécité face à la littérature contemporaine $»^{10}$. «Jamais il ne s'intéressa spontanément à un jeune talent $\gg^{11}$ : cette idée tirée du texte Postérité à propos de Sainte-Beuve montre, en effet, les objectifs de Mirbeau dans la critique, les objectifs plus ambitieux et, si l'on veut, plus historiques que ceux de la réclame. «Il y a contre nous une force plus forte que la vérité, et qu'on appelle l'Histoire» ${ }^{12}$, écrit Mirbeau dans le texte Une page d'bistoire en 1890.

Étant créateur, l'esprit critique de Mirbeau est sensible aux formes, aux moyens d'expression qui le mènent vers l'essence de l'art. Dans son article Le Rêve, en luttant contre «l'exactitude des romans naturalistes», il nous présente sa vision de la fonction de l'art:

L'art n'est point fait pour nous apprendre quelque chose; il est fait pour nous émouvoir, pour nous bercer, pour nous charmer, pour nous faire oublier les réalités brutales et les dégoûts de tous les jours, pour remuer dans l'homme ce qu'il y a de meilleur en lui, ce qu'il y a d'étouffé par la vie, et de délaissé et d'endormi au fond de son être ${ }^{13}$.

(7) O. Mirbeau, Des Artistes, première série, Paris, Flammarion, 1922, p. 167.

(8) Ibid., p. 196.

(9) L'Histoire littéraire des écrivains, sous la direction de V. Debaene, J.-L. Jeannelle, M. Macé et

M. Murat, Paris, Presses de l'Université Paris-Sorbonne, 2013, p. 20.

(10) Ibid.

(11) O. Mirbeau, Les Écrivains, première série, Paris, Flammarion, 1926, pp. 99-108.

(12) Ibid., pp. 193-202.

(13) Ibid., pp. 21-27. 
L'historicisme de l'époque, sans négliger l'influence de l'étude sur l'évolution littéraire de Brunetière, a sans doute subi une mutation importante avec Mirbeau. En tant que critique, il suit d'une manière journalière les auteurs contemporains, il les analyse et il les juge, mais en même temps il les voit et les place dans une échelle diachronique en tant qu'historien. Tout ce que nous appelons la méthode externe, c'est la cible de son intérêt d'historien - les amitiés, les institutions, l'opinion publique, les jugements des autres critiques. Les éléments de la méthode interne - le style, le langage, la forme, la couleur - forment avec les éléments extratextuels une vue complète sur l'œuvre, l'auteur, le genre. Dans la critique mirbellienne on retrouve selon les termes de Bourdieu le champ littéraire dans le champ du pouvoir, et c'est justement le champ des historiens littéraires par excellence. L'art, la littérature intéresse Mirbeau dans sa totalité, dans son intégralité. Avec le regard critique, tourné vers le texte mais aussi vers les forces extratextuelles, il révèle une méthode critique originale et singulière, située entre les méthodes connues en France à l'époque, entre le positivisme et l'impressionnisme, entre le journalisme et l'académisme. En évitant les systèmes théoriques aussi bien que le dilettantisme de la critique journalistique, Mirbeau profite de tout ce que la critique française offre au XIX ${ }^{e}$ siècle.

\section{Le Jugement de Mirbeau}

Si l'on met à part la multitude des textes consacrés aux phénomènes sociologique de l'art et si l'on jette le regard sur les analyses critiques d'Octave Mirbeau, on découvre au début un procédé littéraire, presque balzacien. Séduit par l'atmosphère, l'ambiance, l'odeur et la saveur de la vie de l'auteur, le lecteur est intégré dans le monde mirbellien où le personnage principal de ces petits drames, l'artiste, se présente vivant, réel, soit génial, soit médiocre. Le registre de Mirbeau est varié, du sérieux au parodique, du ludique au familier, mais l'approche reste plus ou moins la même. Présenter la vie de l'artiste dans le contexte social, montrer l'œuvre et finalement, une des fonctions nécessaires de la critique, porter un jugement avec la prévision d'un historien, toutes ces missions critiques fonctionnent bien dans les articles de Mirbeau.

Les notions clés de ses critiques sont l'originalité, le génie, le chef-d'œuvre, et parallèlement la banalité, la médiocrité, la platitude comme les dangers les plus graves de l'art. Ses jugements peuvent être relatifs à l'artiste. «Doué, original et puissant» (Knut Hamsun), «le grand talent», «le génie» (Émile Zola), «un grand artiste» (Auguste Rodin), «très puissant artiste» (Claude Monet), «un artiste très exceptionnel, très troublant» (Paul Gauguin), «un chercheur éternel» (Camille Pissarro), «admirable et rare artiste» pour Camille Claudel (Mannequins et critiques) ou «le favori de la médiocrité» pour Ludovic Halévy (Academiana).

Le critique Mirbeau exprime les jugements sur les genres littéraires et les œuvres également. Pour le conte, il parle de «ce genre délicat et difficile», et encore dans l'article Les conteurs il ajoute: «le conte est un genre charmant et très français, qui a doté notre patrimoine littéraire de beaucoup de chefs-d'œuvre» ${ }^{14}$. Toujours conscient de l'histoire littéraire, Mirbeau évalue l'œuvre des auteurs établis et canonisés au même niveau que celle des opprimés, des marginaux, des inconnus, des jeunes. Pour le Portrait de Dorian Gray il note: «un art brillant et précieux, en même temps qu'une 
intelligence profonde et rare» au moment où Oscar Wilde subit sa peine sociale et morale, en 1895. «J'avoue que ce livre n'est point écrit pour les jeunes filles et qu'il exhale cette odeur impure dont parle M. Marcel Prévost» ${ }^{15}$, réplique Mirbeau aux accusateurs. Avec les mots de Wilde lui-même pour conclure la question de la morale dans la littérature, il écrit: «Un livre n’est point moral ou immoral, il est bien ou mal écrit» ${ }^{16}$.

Les critiques de Mirbeau qui sont en même temps les défenses et les combats, sortent du domaine esthétique et elles deviennent ainsi des combats éthiques. L'antidogmatisme de sa méthode lui permet de chercher l'originalité et de nouvelles formes en tant que créateur et en tant que critique.

\section{Le travail de canonisateur}

Dans le travail critique de Mirbeau, on a déjà souligné la conscience historique de sa méthode. Cette part de sa longue activité de critique, tournée vers les auteurs contemporains, a pour conséquence l'engagement (le mot convient bien à Mirbeau) dans la hiérarchie littéraire. Il reprend le devoir des académiciens et des institutions, ses principaux ennemis, le devoir de cataloguer les auteurs - ce que les historiens font depuis toujours, des anciens bibliothécaires d'Alexandrie aux professeurs d'université à la fin du XIX ${ }^{e}$ siècle. Par ses critiques, Mirbeau élargit le domaine de la lutte, il devient lui-même un canonisateur.

La classification de Thibaudet, évoquée au début du texte, montre que la méthode d'Octave Mirbeau couvre tous les domaines de la critique: elle est évidemment journalistique, elle reflète aussi le créateur; mais il est un peu surprenant que cette méthode pénètre également dans l'univers clos de la critique des professeurs qui rédigent des histoires et canonisent les artistes. Difficilement classable parmi les méthodes connues, la méthode critique de Mirbeau témoigne d'une mutation extraordinaire des connaissances théoriques et critiques au XIX ${ }^{\mathrm{e}}$ siècle, avec des résultats exceptionnels, avant tout dans l'ordre de la hiérarchie artistique.

L'histoire de la littérature est le résultat de regards successifs de plusieurs générations sur la tradition. Aujourd'hui, un siècle après, avec une bibliothèque exhaustive des histoires littéraires, des manuels, avec des programmes universitaires, on peut facilement voir la place et le statut des auteurs analysés par Octave Mirbeau. On peut aussi vérifier les jugements critiques et le résultat de ses combats.

La découverte d'Auguste Rodin, par exemple, aujourd'hui reconnu comme le plus grand sculpteur français, est due à Mirbeau, qui était conscient et fier de son intuition. En 1885, il écrit qu' «Auguste Rodin est à peu près inconnu» ${ }^{17}$. Après plusieurs articles sur Rodin, dans le texte de 1897 Kariste parle, Mirbeau est tout à fait sûr de son évaluation:

Tu te rappelles, hein? Tu te rappelles que nous fûmes les seuls - les seuls, ah! il faut le dire - à nous extasier devant ce morceau unique, ébouriffant de modelé, de mouvement et de couleur? Toute la critique défila devant et ne s'arrêta point. Tu te rappelles? Elle crut que Rodin, pressé de réunir son exposition, avait mis là un plâtre quelconque, ébauche vague, informe

(15) O. Mirbeau, Les Écrivains cit., pp. 45-52.

(16) Ibid.

(17) ID., Des Artistes cit., p. 13. 
esquisse et qui ne comptait pas ... pour faire nombre. Car, c'est cela, elle crut que c'était une ébauche, la critique... Tu te rappelles ${ }^{18}$ ?

Les jugements de Mirbeau ou de son interlocuteur fictionnel Kariste participent encore plus à la reconnaissance du talent de Camille Claudel, cette lacune honteuse de l'art français. Mais, reprenons les mots de Mirbeau: l'Histoire est plus forte que la vérité. Octave Mirbeau est un critique rare qui savait identifier le génie de la jeune artiste alors inconnue. «Elle a du génie», répétait Mirbeau devant ses sculptures. Il reproche à la critique les phrases conventionnelles concernant cette «merveilleuse» artiste. Toujours dans le domaine des valeurs éternelles de l'histoire de l'art, Mirbeau prévoit le destin de Camille Claudel en 1897:

Si nous étions dans une autre époque [...] une femme, comme Mlle Claudel, on la couvrirait d'honneurs et d'argent... Car de tels artistes, c'est la gloire d'une époque... ce qui reste de plus grand, de plus pur, d'une époque... Oui... Eh bien! Que fait-on de cette énergie, de cette volonté, de cet hérö̈sme d'art?... Rien... rien... rien... ${ }^{19}$.

La place dans les histoires de l'art est préparée tout d'abord par les critiques contemporains. La réception ultérieure, les revalorisations, les travaux scientifiques, les recherches universitaires viennent après. La critique prépare la documentation et les matériaux pour l'histoire. Les articles journalistiques réguliers de Mirbeau permettent une réception continue et nous avons aujourd'hui, grâce avant tout à la publication de ses textes rassemblés sur le site www.mirbeau.org, le témoignage d'une activité critique inouïe. La fameuse période de la Belle Époque s'ouvre aux lecteurs, qui assistent au processus même des premiers accueils des artistes, suivant un vrai work in progress. Mirbeau déplore la mort précoce de Vincent Van Gogh et l'incompréhension dont il est victime de son vivant. Il est en colère à cause de l'anonymat de Gauguin. Pour Claude Monet, il juge «qu'il n'a été l'élève de personne» ${ }^{20}$. Ses défenses sont aussi bien professionnelles que personnelles, éthiques, finalement émotionnelles, «passionnées» ${ }^{21}$. Christian Limousin est parmi ceux qui ont identifié cet aspect de la critique mirbellienne:

En art, tout commence pour Mirbeau par la sensation. Tout est chez lui éminemment physique. Son hypersensibilité (qui se retrouve dans ses personnages de roman) fait que les chefs-d'œuvre lui donnent le frisson, le bouleversent aux larmes ${ }^{22}$.

Des artistes favoris d'Octave Mirbeau, ou qui ont provoqué en lui ce «frisson», occupent aujourd'hui une place remarquable dans les histoires de l'art ou de la littérature en tant qu'auteurs canoniques (Rodin, C. Claudel, Pissarro, Monet); ceux qu'il a ridiculisés sont restés dans l'obscurité de l'histoire.

(18) Ibid., deuxième série, p. 14.

(19) Ibid., pp. 20-21.

(20) Ibid., première série, p. 90.

(21) P. Michel, Les Combats d'Octave Mirbeau cit., p. 90.

(22) C. Limousin, La Critique d'art de Mirbeau: de "l'âge de l'buile diluvienne" au règne de l'artiste de génie, «Cahiers Octave Mirbeau», 1, 1994, pp. 11-41. 


\section{Le cas de Maeterlinck}

Parmi les découvertes littéraires d'Octave Mirbeau Maurice Maeterlinck occupe une place exceptionnelle. L'auteur belge, qui va bientôt devenir mondialement reconnu, de la Russie aux États Unis, récompensé par le prix Nobel en 1911, l'auteur joué dans toute Europe, Maeterlinck doit sans doute à notre critique toute sa gloire. Même la réception ultérieure de l'écrivain, en France et à l'étranger, se réfêre à l'article fameux que Mirbeau lui consacre le 24 août 1890.

Pourquoi ce texte est-il fameux? Parce que dans un même texte il y a tout l'art de Maeterlinck, mais en même temps il y a tout ce qu'on a déjà défini comme la méthode critique de Mirbeau. Il cerne l'esthétique de l'auteur belge et, probablement, il la dirige dans les choix poétiques futurs de Maeterlinck. Avec ses jugements forts, sa connaissance de la littérature contemporaine, Mirbeau jalonne l'histoire littéraire d'une nouvelle esthétique du drame et lance un auteur. La Princesse Maleine, «un drame écrit pour un théâtre de fantoches», dans l'alchimie critique de Mirbeau est promu à un chef-d'œuvre:

[...] un admirable, et pur, et éternel chef-d'œuvre qui suffit à immortaliser un nom et à faire bénir ce nom par tous les affamés du beau et du grand; un chef-d'œuvre comme les artistes honnêtes et tourmentés, parfois, aux heures d'enthousiasme, ont rêvé d'en écrire un, et comme ils n'en ont écrit aucun jusqu'ici2 ${ }^{23}$.

La comparaison avec Shakespeare, la reconnaissance du nouveau langage dramatique, le tragique du drame, les personnages, la poésie, l'effet produit - l'effroi total, ce sont les appréciations que la critique va reprendre, traduire et répéter sans cesse dans la réception de Maeterlinck. Mirbeau, ce chercheur de nouvelles formes affirme que: «Pour arriver à cette impression d'effroi total, M. Maurice Maeterlinck n'emploie aucun des moyens en usage dans le théâtre ${ }^{24}$. On ne peut pas négliger son admiration, toute intime et sensuelle, qui se dévoile comme le procédé mirbellien spécifique:

J'ai longtemps hésité avant de parler de La Princesse Maleine. La laisser dans son obscurité scrupuleuse, ne pas l'exposer, si frêle, si chaste, si adorablement belle, aux brutalités de la foule, aux ricanements des gens d'esprit, être quelques-uns seulement à en jouir, il me semblait que cela valait mieux ainsi ${ }^{25}$.

Finalement, pour conclure l'article, encore un procédé habituel de la méthode de Mirbeau: le reproche à la critique du silence qui accompagne le livre.

L'exemple de l'écho des jugements de Mirbeau est la critique de la presse de Belgrade après la première de La Princesse Maleine au Théâtre national en 1891. Dans un article paru dans la revue «Odjek» («Écho», en français), le critique impressionniste M. S. transmet l'admiration, la joie, l'émoi que le drame mis en scène provoque. Après la description de l'intrigue, il répète la comparaison avec Shakespeare et son Hamlet; le critique serbe souligne ensuite la nouveauté du langage poétique de Maeterlinck, qui est plus naturel sur la scène que les tirades du théâtre classique. Les jugements de Mirbeau restent le guide dans la réception très étonnante de Maeterlinck en Serbie, juste libérée des Ottomans - les cinq pièces (La Princesse Maleine, L'Intruse, Monna

(23) O. MirbeAu, Les Écrivains, première série cit., pp. 174-184.

(24) Ibid.

(25) Ibid. 
Vanna, L'Intérieur et La Mort de Tintagiles), ont été six fois mises en scène à Belgrade et à Novi Sad dans la période 1891-1904².

Comme avec Rodin, Mirbeau est conscient de sa découverte, il présente l'œuvre de Maeterlinck régulièrement et les résonances de son article révélateur également (par exemple Propos belges, le 26 septembre 1890), et il continue à défendre le talent de l'auteur belge. Monna Vanna («un autre chef-d'œuvre»), Les Aveugles, Pelléas et Mélisande, la publication des livres, les mises en scène, toute l'œuvre de Maeterlinck met en joie Mirbeau qui comme un critique intime s'adresse finalement au Flamand dans l'article de 1902:

Et je n'ai pas voulu autre chose, mon cher Maeterlinck, au seuil de cette semaine, qui sera toute pleine de votre nom, et tout embellie de vos œuvres, je n'ai pas voulu autre chose que de saluer d'un mot amical et fervent le Temple Enseveli, que vous m'avez dédié, Pelléas et Mélisande, à qui, autrefois, vous m'aviez fait la grande joie d'associer mon nom, et cette rouge et superbe Monna Vanna, que vous m'avez permis de lire avant les autres, et qui, si le culte de la beauté existe encore, chez nous, sera acclamée, frénétiquement, comme une victoire.

Voilà une grande et noble et triple joie que nous vous devons, en attendant toutes celles que votre génie nous réserve, pour l'avenir... celui qu'il n'est point besoin d'aller demander aux magiciennes de la main, des cartes et du marc de café... ${ }^{27}$.

L'avenir est toujours dans les pensées d'Octave Mirbeau.

$\mathrm{Au}$ nom du Nobel belge, on peut ajouter les exemples littéraires nombreux, dont les poétiques sont tout à fait différentes, de Rodenbach à Maupassant, de Zola à Valery Larbaud, sans oublier la découverte du roman russe. Dans la tradition, récente quand même, en tant qu'historien, il choisit ses canons: Baudelaire, Balzac, Hugo, Flaubert.

\section{Le canonisateur non canonisé}

Le canon, notion d'origine religieuse, permet des querelles, des inclusions et des exclusions. Cet article l'accepte comme la notion de la théorie littéraire issue des études poststructuralistes pour lesquelles «la querelle du canon» mérite une place centrale dans leurs luttes contre les valeurs traditionnelles. «Cette question du canon n'est pas par hasard au cœur du conflit opposant multiculturalistes radicaux et conservateurs au pouvoir» ${ }^{28}$. Aujourd'hui, quand les notions canonisation et textes canoniques sont courants et fréquents dans les études littéraires, nous introduisons le mot canonisateur, avec une allusion explicite au discours religieux, pour souligner le talent et l'audace d'Octave Mirbeau dans l'évaluation des contemporains.

Il est absurde d'avoir les auteurs que Mirbeau a consacrés dans les histoires de la littérature et de l'art, avec ses jugements, mais sans Mirbeau lui-même. Pourquoi l'avenir, qui a gardé "les artistes de Mirbeau", a-t-il oublié le critique canonisateur? Les histoires de l'art et de la littérature ont repris ses jugements, mais le nom d'Octave Mirbeau critique est presque inexistant dans les précis historiques. Christian

(26) Sur la réception de M. Maeterlinck en Serbie et au Monténégro, voir: M. DJukIC, Raznovrsne interpretacije u recepciji Mauricea Maeterlincka u Srbiji i Crnoj Gori, «Knjizevna smotra», vol. 180, n. 2, pp. $145-152$.

(27) O. Mirbeau, Les Écrivains, deuxième série cit., pp. 253-259.

(28) F. Cusset, French Theory, Paris, La Découverte, 2005, p. 180. 
Limousin montre la «réapparition progressive d'un critique d'art ${ }^{29}$, grâce avant tout à l'activité exceptionnelle de Pierre Michel. Mais les histoires de la littérature taisent encore son nom. Dans la Littérature française, publiée en 2007 sous la direction de Jean Yves Tadié, le critique Mirbeau n'est mentionné que dans le texte consacré au théâtre symboliste.

Mirbeau reste en marges des manuels de la critique artistique et littéraire. S'il est mentionné, il se trouve dans la case de la critique journalistique. On découvre son nom en général grâce aux artistes qu'il a suivis par ses articles. Les spécialistes d'Octave Mirbeau, avant tous Pierre Michel et l'activité de la Société Octave Mirbeau, ont déjà réalisé un véritable exploit par la publication des textes critiques qui sont en grande partie mis en ligne. Ce fait ouvre la possibilité aux chercheurs de découvrir et d'étudier un artiste original et un critique singulier. Cependant, Samuel Lair atteste, après la publication des Combats littéraires, que «la parution d'une œuvre majeure est à l'abri d'un silence aussi assourdissant, de la part de la critique» ${ }^{30}$. La célébration du centenaire de la mort d'Octave Mirbeau distribue la vérité au monde d'une manière presque miraculeuse. Est-ce que cela sera suffisant pour que Mirbeau obtienne sa place fixe dans l'histoire de la littérature? Il la mérite certainement. L'héritage riche de la pensée française dans le domaine de la critique artistique, absorbé dans la méthode de Mirbeau, nous présente un esprit ouvert, libre, créateur qui forme une méthode critique particulière et unique. L'innovation dans les registres, dans les approches, dans la forme - la critique dialoguée par exemple, nous montre un grand novateur, un juge audacieux et un canonisateur crédible.

MARJANA DJUKIC

Université du Monténégro

(29) C. Limousin, La Critique d'art de Mirbeau: de "l'âge de l'buile diluvienne" au règne de l'artiste de génie cit., p. 11.

(30) S. Lair, Les Combats littéraires d'Octave Mirbeau, «Cahiers Octave Mirbeau», 14, 2007, p. 174. 